\title{
A Variation of Reporting Verbs in Thai EFL Graduate Student's Academic Writing: A Corpus-Based Study
}

\author{
Anchalee Veerachaisantikul $^{1}$, Boonchai Srisassawatkul ${ }^{2}$ and Sukanya Chootarut $^{3}$ \\ ${ }^{1,2,3}$ English for International Communication Department \\ Rajamangala University of Technology Isan, Thailand
}

\begin{abstract}
This paper aimed to explore the impact of English for Academic Purposes course on the development of academic writing of EFL graduate students in mechanical engineering at the Rajamangala University of Technology Isan (RMUTI). In academic writing at the university, students need to use various sources and refer to the research of others. One of important instruments that used to talk or cite on other people's work is reporting verb. However, EFL graduate students are frequently insufficient to use it properly. Consequently, the present study aimed to investigate 1) which reporting verbs are most frequently used by EFL graduate students in their research articles, and 2) which group of reporting verbs do most of them use in research articles. In order to achieve the purposes of the study, a corpus of 30 research articles written by EFL graduate students in mechanical engineering were selected and investigated. Additionally, this study adopted the categorization of Francis et al. (1996) to classify reporting verbs. The results revealed that the top five high frequency reporting verbs were "predict", "show", "agree", "observe", and "direct". After forming the reporting groups, the findings also demonstrated that the highest percentage of reporting verb groups of this study were the ARGUE verb group (30\%) followed by FIND (20\%), THINK (18\%), SHOW (18\%), and ADD verb group (4\%) respectively.
\end{abstract}

Keywords: reporting verb, EFL graduate students, corpus-base study

\section{Introduction}

In graduate-level writing, being able to write various types of academic in English for example research proposals, conference papers, journal articles, or theses and dissertation has been of an important ability for graduate students in many disciplines (Casanave, 2002; Paltridge, 1997). Although, there are demanding on writing academic papers in English as a Second/Foreign Language (ESL/EFL) learners, it could be discouraged them. Since, students at different education levels feel concerned to write in varying degrees. One of troubled task is research article.

Research articles (RAs) is one genre of academic writing which regarded as effective means of transferring and promoting knowledge within the academic community (Flowerdew, 1999). Reading and writing research articles are essential to members of academic community at the more advanced level of education such graduate students. In writing research article, they require to use various sources and refer to the others' research. Moreover, they have to promote and discuss previous studies, make references to claim in order to design their research spaces (Swales, 1990 and Zhang, 2008). One of important instrument for using to discuss or report on other people's work is reporting verb.

Reporting verb is a noticeable semantic word in academic writing using in general to talk or present the research of others and to state the knowledge and understanding or attitude to the sources. Since, it provides a scope of purposes, reveals the writer's familiarity with the field, as tools to talk into and to probe the writer's work in the greater context (Bavelas, 1978, Gilbert, 1977, and Myers, 1990). For this reason, reporting verb is a crucial academic skill for being brief the entire knowledge or outcome of people's work. On the other hand, language teachers and students usually disregard the importance and complication of its in grammar books and language textbooks as it is noticed as an ordinary linguistic which not need to characterize (Zhang, 2008). Besides, there are some researchers quoted that non-native writers have more or less difficulties in selecting reporting verbs in their academic writing (Bruce, 1989, Granger, 1993, Pickard, 1995, Thompson and Ye, 1999). This confirmed that it is essential tool and has to be used with consideration in academic writing. 
In Thailand, English is taught as English as a Foreign Language (EFL) thus the current study is mostly relevant to the complications and matter in dealing with reporting verb of EFL graduate students. Additionally, there is poor research on reporting verb in Thailand thus the current study is aimed to reach the purposes as follow:

1. To profile the most frequently used reporting verbs in research articles written by EFL graduate students in mechanical engineering field.

2. To classify the most frequently group of reporting verbs in research articles of EFL graduate students in mechanical engineering field.

\section{Method}

\subsection{Corpora}

The compilation of the current study was gathered from scientific articles belonging to one principal area of scientific knowledge: the field of engineering. The corpora consisted of a corpus of thirty research articles written by EFL graduate students. The amount of items selected in the corpus was 451,236 running words. The thirty research articles written by EFL graduate students were all compiled from the international journal and the proceeding of international conference in the specific field of Mechanic Engineering during 2013 to 2015. The section organization of their research article were followed a general format in all seven main sections (Abstract, Introduction, Literature Review, Method, Results, and Discussion and Conclusion, and Reference). In order to meet the research purposes, the reporting verb list of the corpus represented the Abstract, Introduction, Methodology, Results, and Discussion section followed by Swales (1999). Table 1 gives an overview of the RAs Corpus used for the EFL graduate students.

\begin{tabular}{cc} 
TABLE I: An Overview of the RAs Corpus \\
\hline RAs Corpus \\
\hline Running Words & 451,236 \\
Sentences & 3,431 \\
Mean sentence length & 19.11 \\
Frequency of Reporting verb & 917 \\
\% of Reporting verb Coverage & 0.20
\end{tabular}

Table 1 contains information on an overview of the RAs Corpus. The number of token words (running words) were 451,236 words and the mean of sentence length was 19.11. As it is seen, there was a total of reporting verb found in this corpus were 917 occurrences that for $0.20 \%$ of the whole corpus.

\subsection{Procedure and Data Collection}

To reach the purposes of the study, we gathered 30 English research articles written by EFL graduate students in order to form a list of reporting verb and group of reporting verbs most of them use in their research articles. As a result, there are two main procedures of the analysis that composed of collecting reporting verb frequency and classifying their groups. The concordancing software called "WordSmith Tool Version 6" (Scott, 2012) was selected for this study. This is an integrated suite of program for making the list of reporting verb. Since, all research projects were in MS Word (.doc) and PDF file then we converted them into text file (*txt.) in order to apply with the concordancing software. Firstly, the Wordlist tool was used to make the word frequency lists of research articles. This tool of software program offered both alphabetical and frequency order of the reporting verb. Then, the most frequently occurring reporting verbs were obtained then we classified the groups of reporting verbs used in the RAs Corpus.

\subsection{Data Analysis}

To respond the first research purpose that is about the frequency of reporting verbs in research articles of EFL graduate students, an attempt is made to select reporting verb words that frequently appeared in the complied corpus of this study. The list is obtained from the help of the concordancing software "WordSmith Tool Version 6". Additionally, the second research purpose focused on exploring the groups of reporting verb 
found in the RAs Corpus. After obtaining the list of the most frequently reporting verb, we classified the verb groups on the basis of Francis et al. (1996) as follows:

1. ARGUE verbs are concerned with speaking, writing and other forms of communication, e.g. argue, suggest, point out, write, conclude, claim, add, maintain, propose, imply, and mention.

2. THINK verbs are concerned with thinking including having a belief; knowing, understanding, hoping, fearing, e.g. think, assume, feel, hold, and believe.

3. SHOW verbs are concerned with indicating a fact or situation, e.g. show, demonstrate, and reveal.

4. FIND verbs are concerned with coming to know or think something, find, observe, discover, and indicate.

5. ADD verbs are concerned with the relationship of something that is said or written to something else that has been said or written, e.g. add, repeat, and verify.

\section{Results}

The present study concentrated on the frequency, coverage, and distribution of reporting verb in the RP Corpus. Consequently, a corpus of 451,236 running words from EFL graduate students' research articles has been applied. This section is structured to answer the two posted research purposes.

Research Purpose 1: To profile the most frequently used reporting verbs in research articles written by EFL graduate students in mechanical engineering field.

TABLE II: The Most Frequently Reporting Verbs of the RAs Corpus

\begin{tabular}{|c|c|c|c|c|c|}
\hline Rank & Word & $\mathbf{F}$ & Rank & Word & $\mathbf{F}$ \\
\hline 1 & predict & 128 & 26 & discover & 11 \\
\hline 2 & show & 108 & 27 & reflect & 10 \\
\hline 3 & agree & 60 & 28 & hypothesize & 10 \\
\hline 4 & observe & 43 & 29 & recommend & 10 \\
\hline 5 & direct & 39 & 30 & state & 10 \\
\hline 6 & report & 33 & 31 & explain & 9 \\
\hline 7 & illustrate & 28 & 32 & imply & 9 \\
\hline 8 & reason & 28 & 33 & emphasize & 9 \\
\hline 9 & consider & 27 & 34 & indicate & 8 \\
\hline 10 & determine & 26 & 35 & conclude & 8 \\
\hline 11 & order & 24 & 36 & claim & 7 \\
\hline 12 & mention & 24 & 37 & say & 6 \\
\hline 13 & mean & 24 & 38 & expect & 6 \\
\hline 14 & accept & 20 & 39 & note & 5 \\
\hline 15 & assume & 18 & 40 & resolve & 5 \\
\hline 16 & denote & 18 & 41 & signal & 5 \\
\hline 17 & understand & 17 & 42 & maintain & 4 \\
\hline 18 & establish & 16 & 43 & prove & 3 \\
\hline 19 & confirm & 13 & 44 & add & 3 \\
\hline 20 & reveal & 13 & 45 & suppose & 2 \\
\hline 21 & know & 12 & 46 & demonstrate & 2 \\
\hline 22 & find & 12 & 47 & decide & 2 \\
\hline 23 & find out & 12 & 48 & deduce & 2 \\
\hline 24 & suggest & 11 & 49 & repeat & 2 \\
\hline 25 & calculate & 11 & 50 & verify & 2 \\
\hline
\end{tabular}


From the data shown in Table 2, it provided the most prevalent reporting verbs in the RAs Corpus. The total of frequency of occurrences in this table was calculated from the $1^{\text {st }}$ rank to $50^{\text {th }}$ rank and there was a total of 917 occurrences. According to the list, the top five high frequency words were "predict" 128 times, "show" 108 times, "agree" 60 times, "observe" 43 times, and "direct" 39 times.

Research Purpose 2: To classify the most frequently group of reporting verbs in research articles of EFL graduate students in mechanical engineering field.

After obtaining the top most frequently reporting verbs, we classified the verb groups by adapting from Francis et al. (1996). According to Table 3, the highest percentage of reporting verbs groups for the RAs Corpus refer to the ARGUE verb group (30\%). In addition, the findings also presented that EFL graduate students tend to use FIND $(20 \%$,) as their second preference and followed by THINK verbs (18\%) SHOW (18\%), and ADD (4\%) verb group respectively as shown below.

TABLE III: The Groups of Reporting Verbs of the RAs Corpus

\begin{tabular}{ll|ll|ll|ll|l}
\hline \multicolumn{2}{c|}{ ARGUE } & \multicolumn{2}{c|}{ THINK } & \multicolumn{2}{c|}{ SHOW } & \multicolumn{2}{c}{ FIND } & \multicolumn{1}{c}{ ADD } \\
\hline accept & claim & assume & consider & confirm & demonstrate & calculate & conclude & agree \\
direct & emphasize & expect & know & denote & illustrate & decide & deduce & add \\
explain & hypothesize & resolve & maintain & indicate & mean & determine & discover & repeat \\
imply & note & mention & suppose & prove & reveal & establish & find & verify \\
order & predict & understand & & show & & observe & find out \\
reason & recommend & & & & & & \\
reflect & report & & & & & & & \\
reveal & say & & & & & & & \\
state & suggest & & & & & & & \\
\hline
\end{tabular}

\section{Discussion}

According to the findings, the current study purposes to rank the frequency of reporting verb used by EFL graduate students and which groups of reporting verb most of them applied in their research articles. When investigating the frequency of reporting verb, it was seen (Table 2) that the percent of reporting verb coverage was only $0.20 \%$ of the whole text. It was obvious that reporting verbs appeared less often than the academic or general words as the function of them are specific purpose. As theirs characteristics, we usually use them to refer to the academic writer's previous studies with ideas, research outcomes or findings, and research activities of previous research agents whereas the academic or general words use to write or describe the whole paper. Moreover, when classifying the groups of reporting verb, it was found (Table 3) that EFL graduate students tended to use the ARGUE verb group in the highest percentage (30\%). FIND verb was used in the second rank (20\%), THINK (18\%), SHOW (18\%), and ADD verb group was used in the lowest rank (4\%) respectively. These findings were close to Hyland (2002) that writer or researchers in mechanical engineering preferred to use "show" and "report" than other verbs. Yet, the findings of the current study were quite different from Hyland's work in the ranking. The "show" and "report of Hyland's work was at the second and third rank; however, in the current study the "show" was at the second rank and "report" was the sixth rank.

\section{Conclusion}

As Manan and Noor (2014) suggested that the importance and the effect of reporting verb, they could have on how writers are perceived teaching of these verbs and how they are used an important part in research methodology and academic writing courses. Consequently, the current study aimed to study most frequently used reporting verbs in researcher articles of EFL graduate students and groups of reporting verb do most of them use in their research articles. Furthermore, the findings exhibited the total number of research articles, the number of tokens or running words, and the total of occurrences.

In this study, we compiled 30 research articles of EFL graduate students and the findings showed the tokens or running words of the RAs Corpus were 451,236 words. Also, the findings presented that the five top most 
prevalent reporting verbs in the RAs Corpus were "predict" 128 times, "show" 108 times, "agree" 60 times, "observe" 43 times, and "direct" 39 times respectively.

After classifying the groups of reporting verb, the findings presented that EFL graduate students tended to use the ARGUE verb group most (30\%). The results also presented that THINK verbs $(19.36 \%)$ as their second preference and followed by SHOW (12.9\%), FIND (12.9\%), and ADD (6.45\%) verb group respectively.

To sum up, this study might be provided the useful information on the differences of reporting verb for academic writers. Although, a research article has the standard four-section formats: Introduction, Methods, Results, and Discussion, the linguistics characteristics such as reporting verb in these sections may vary in different fields. Furthermore, this study attempted to help in order to raise the writer's awareness in selecting reporting verb in research articles or academic writing. Theoretically, it is a creation to study what evidentially can do for the language users other than indication the information source. In addition, the findings of this study have some implications for graduate students, graduate advisors and English for Academic Purposes (EAP) professionals. It may be a foundation for the future researches and provide orientation for further studies. As there are more areas to be further studied, for example, the functions of other evidential types, the genre convention, and evidential use in other genres. Besides, an evidential use across genres in different cultures may vary and it is regarded to be an interesting topic in significant study.

\section{References}

[1] Bevalas, J. B. (1978). The social psychology of citations. Canadian Psychological Review, vol. 19, pp.158-63. https://doi.org/10.1037/h0081472

[2] Bruce, N. J. (1989). The roles of analysis and the conceptual matrix in a process approach to teaching academic study and communication skills. In V. Bickley, ed. Language Teaching and learning Styles within and across Cultures. Hong Kong: Hong Kong Education Press

[3] Casanave, C. P. (2002). Writing games: Multicultural case studies of academic literacies in higher education. Mahwah, NJ: Lawrence Erlbaum.

[4] Francis,G., Huston, S., \& Manning, E. (1996). Collins Cobuild Grammar Pattern 1: Verbs. London: Harper Collins.

[5] Gilbert, N. G. (1977). Referencing as persuasion. Social Studies of Science, vol. 7. pp. 113-22. https://doi.org/10.1177/030631277700700112

[6] Granger, S., 1993. 'New insights into the learner lexicon: a preliminary report from the International Corpus of English'. In L. Flowerdew, \& K. Tong, eds. Entering text Hong Kong: The Hong Kong University of Science and Technology

[7] Hyland, K. (2002). Activity and evaluation: reporting practices in academic writing. In J. Flowerdew (Ed.), Academic discourse. pp. 115-130. London: Longman.

[8] Manan, N. A. \& Noor, N.M. (2014). Analysis of Reporting Verbs in Master's Theses. Procedia - Social and Behavioral Sciences, vol. 134. pp. 140-145. https://doi.org/10.1016/j.sbspro.2014.04.232

[9] Myers, G., (1990). Writing Biology: Texts in the social construction of scientific knowledge. Madison: University of Wisconsin Press.

[10] Paltridge, B. (1997). Thesis and dissertation writing: Preparing ESL students for research. English for Specific Purposes, vol. 16(1), pp. 61-70. Available: http://dx.doi.org/10.1016/S0889-4906(96)00028-2

[11] Pickard, V. (1995). Citing previous writers: what can we say instead of 'say'? Hong Kong Papers in Linguistics and Language Teaching, vol. 18, pp.89-102.

[12] Scott, M. (2012). WordSmith Tools version 6. Stroud: Lexical Analysis Software.

[13] Swales, J. (1999). Informal elements in English academic writing: threats or opportunities for advanced non-native speakers?' in Candlin', C. and Hyland, K. (Eds.) Writing: texts, processes and practices. London, Longman

[14] Thompson, G. \& Ye, Y. (1991). Evaluation in the reporting verbs used in academic papers. Applied Linguistics, vol. 12(4), pp.365-82.

https://doi.org/10.1093/applin/12.4.365 